\title{
Vitamin D Through Balancing Gut Microbiota to Improve Non-alcoholic Steatohepatitis (Nash): A Study Protocol for a Randomized Clinical Trial
}

\section{Yilan Zeng}

Public Health and Clinical Center of Chengdu

Dongxia Luo

Public Health and Clinical Center of Chengdu

\section{Li Wang}

Public Health and Clinical Center of Chengdu

Mei Luo

Public Health and Clinical Center of Chengdu

\section{Chuangjie Mao}

Public Health and Clinical Center of Chengdu

\section{Li Zhu}

Public Health and Clinical Center of Chengdu

\section{Lisha Pan}

Sichuan University

\section{Siya $\mathrm{Xu}$}

Sichuan University

\section{Pengfei Wu}

Sichuan University

\section{Rong $\mathrm{Hu}$}

Public Health and Clinical Center of Chengdu

\section{Bei Wu}

Public Health and Clinical Center of Chengdu

Hong Jin

Public Health and Clinical Center of Chengdu

\section{Dafeng Liu}

Public Health and Clinical Center of Chengdu

Jun Lin

Public Health and Clinical Center of Chengdu

\section{Qingfeng Li}

Public Health and Clinical Center of Chengdu

\section{Ruofei Zhang}


Sichuan University

\section{Mazen Noureddin}

Cedars-Sinai Medical Center

\section{Stephen Pandol}

Cedars-Sinai Medical Center

\section{YUAN-PING HAN ( $\square$ hanyp@scu.edu.cn )}

Sichuan University https://orcid.org/0000-0001-6282-7319

\section{Research Article}

Keywords: Vitamin D, NASH, non-alcoholic steatohepatitis, VD2, gut microbiome

Posted Date: September 24th, 2021

DOI: https://doi.org/10.21203/rs.3.rs-868710/v1

License: (c) (i) This work is licensed under a Creative Commons Attribution 4.0 International License. Read Full License 


\section{Abstract}

\section{Background}

Vitamin D deficiency (VDD) is overwhelmingly prevalent worldwide in association with metabolic syndrome (MetS), type-2 diabetes (T2D), and non-alcoholic fatty liver diseases (NAFLD). Whether vitamin D supplement or treatment can improve these metabolic diseases is controversial, due in part to absence of large-scale trials. On the other hand, unbalanced gut microbiome, dysbiosis, plays key roles in these metabolic diseases. Moreover, whether sunlight exposure or vitamin D can determine the gut microbiome is elusive. The present study aims to investigate whether intramuscular administration of high dose of vitamin $\mathrm{D}_{2}$ can improve non-alcoholic steatohepatitis (NASH) through balancing of gut microbiome.

\section{Methods/design}

In a randomized, open-labeled, and parallel-group trial, NASH subjects will be enrolled and randomly allocated to receive ergocalciferol $\left(\mathrm{VD}_{2}\right)$ treatment or not, $\mathrm{n}=80$ for each group. The NASH patients, diagnosed according to the guideline for NAFLD treatment of China, will be given an initial dose of $\mathrm{VD}_{2}$ at 600,000 IU by intramuscular injection followed by additional three administration every other 30 days with total of four injections in 120 days. Anthropometry, liver functions, the metabolic panel will be measured. Fatty liver index will be determined by topological analysis of ultrasound data. Serum 25-hydroxyvitamin D (25(OH)D) and fecal microbiome will be measured. Discussion: This study will determine the efficacy of high dose of $\mathrm{VD}_{2}$ to relieve NASH symptoms and on gut microbiota.

Trial registration

Chinese Clinical Trial Registry, ChiCTR1800017879, registered on Aguste 20 2018.

\section{Background And Rationale}

Non-alcoholic fatty liver diseases (NAFLD) is characterized as excess deposition of fat in the liver, and featured as a progression from simple steatosis (NAFL) to non-alcoholic steatohepatitis (NASH), cirrhosis, and even hepatocellular carcinoma (HCC)[1]. Metabolic disorders including insulin resistance, obesity and dyslipidemia are associated factors of NAFLD. Abdominal obesity, also known as central obesity, is tightly associated with NAFLD. Incidence of NAFLD varies according to geological regions, global latitude, diet habits, aging, and race. The prevalence of NAFLD is constantly increasing worldwide from $15 \%$ in 2005 to $25 \%$ in 2010 [2]. In China, incidence of NAFLD is about $15 \%$ in general population [3]. In the US, NAFLD affects an estimated 64 million Americans. The steatosis is usually the consequence of excessive calorie intake, high fat or high sugar diet, and sedentary life style. But, the prognosis of simple fatty liver to NASH is often driven by the second hits such as hepatic infection, mitochondrial injury, oxidative stress, microbiota changes and others. 
Humans have evolved on the sunlight dependent metabolism, exemplifying as synthesis of vitamin D3 in the skin by sunlight exposure. Through additional two steps of hydroxylation in the liver and kidney, VD3 (cholecalciferol) or plant produced and dietary VD2 (ergocalciferol) are converted to calcitriol, a biological active component of vitamin $D$. Through activation the nuclear vitamin $D$ receptor (VDR), vitamin $D$ signalling is conveying to transcriptional control of the downstream targeting genes for biological functions such as expression of calcium/phosphorus transporter in the small intestinal epithelial cells. The cis elements of vitamin D receptor response called VDRE are widely presented at genome wide scales, which convert genome wide transcription [4]. Emerging findings in recent years uncovered the pleiotropic functions of vitamin D signalling. Generally, it is known that vitamin D can suppress the adaptive immunity such as Th1 response, and promote innate immunity and immune regulation.

Vitamin D deficiency (plasma 25OH-VD $<20 \mathrm{ng} / \mathrm{ml}$ ) and insufficiency (plasma 25OH-VD $<29$ but $>20$ $\mathrm{ng} / \mathrm{ml}$ ) are related to obesity and NAFLD and cirrhosis $[5,6]$. In a large cross-sectional study, vitamin D deficiency was found in association with NAFLD [7]. Another study showed that vitamin D levels are inversely associated with NASH and fibrosis in children with NAFLD [8]. Vitamin D deficiency and insufficiency are common in children with NAFLD [9]. Because vitamin D insufficiency is common in the general population including healthy ones, its association with NAFLD may not be consistent and even controversial [10-13]. Importantly, the correlation studies are unable to define the causal relationship of vitamin D deficiency in NAFLD prognosis. It is possible that the low 25(OH)VD levels could be the consequence of steatosis and the liver injury, rather than as the cause. Thus, clinical intervention on the NAFLD patients with therapeutical dose of vitamin $D$ is an ultimate way to determine the contribution of vitamin $D$ in pathogenesis of NAFLD. High dose vitamin D supplement has been used for clinical studies related to pulmonary tuberculosis, cystic fibrosis, and Crohn's disease [14-16].

\section{Objectives and hypothesis $\{7\}$}

We hypothesize that vitamin $D$ signaling in the small intestine may maintain the innate immunity to prevent bacterial up translocation, and gut eubiosis. The primary goal of this clinical study is to evaluate the potential benefits of high dose vitamin $\mathrm{D}_{2}$ on NASH patients, including improving liver functions and mitigating fatty liver. The secondary goal of this trial is to determine regulation of gut microbiota by vitamin $D$ presumably through induction of intestinal innate immunity.

\section{Trial design $\{8\}$}

This is a randomized parallel controlled trial. The study protocol has been approved by the Medical Ethic Committee of the Public Health and Clinical Center of Chengdu (\# 2018Y001). This clinical trial has been registered in Chinese Clinical Trial (Registration number: ChiCTR1800017879), and available at http://www.chictr.org.cn/showproj.aspx?proj=25274

NASH patients and healthy controls will be recruited in the Public Health and Clinical Center of Chengdu, Sichuan, China. In agreement with the treatment protocol, all participants will sign the Consent Form. Subjects will be randomly assigned into groups. Questionnaires will be completed at the beginning of the 
study, including alcoholic consumption questionnaire, sunlight exposure, and physical activity. Anthropometric parameters including height, weight, BMI will be measured. Table-1 shows the primary and secondary measurements at day $0,30,60,90$ and day 120 .

\section{Methods}

\section{Participants, intervention, and outcomes}

\section{Study setting $\{9\}$}

The trial, including treatment and data collection, will be conducted in the Public Health and Clinical Center of Chengdu, Sichuan Province, China.

\section{Eligibility criteria $\{10\}$}

\section{Inclusion criteria}

Inclusion criteria for NASH subjects of the clinical trial are: (1) Patients with NAFLD as defined by "Guidelines of Prevention and Treatment for Nonalcoholic Fatty Liver Disease" (China, 2018), (2) age at 18-60 years; (3) ultrasonography, showing fatty liver image, and may be further confirmed by computerized tomography (CT) scanning or magnetic resonance imaging (MRI); (4) elevated liver enzymes, plasma ALT $\geq 2$ folds of the normal or/and GGT $\geq 1.5$ folds of the normal; (5) no habit of alcohol drinking (less than 140g/week for men, and 70g/week for women, and less than twice per week); (5) normal serum levels of calcium and phosphate are; (6) voluntarily participating in this study and sign the consent form. The human subjects also have their right to quite the study.

\section{Exclusion criteria}

Exclusion criteria for the NASH trial are as follows: (1) other serious diseases; (2) HIV infection; (3) breastfeeding or pregnant women; (5) drug abuse; (6) using vitamin D within the previous 6 months; (7) weight loss $>5 \%$ within 3 months before the study; (8) diagnoses of alcoholic liver diseases; (9) drug-induced liver diseases, (10) under total parenteral nutrition, hepatolenticular degeneration, autoimmune liver disease; and (11) other serious systemic diseases and mental illness; (12) poor compliance and voluntarily exiting the study.

\section{Interventions}

\section{Intervention description $\{11 \mathrm{a}\}$}

A flow chart of the study protocol is presented in Fig. 1. Briefly, 160 human subjects diagnosed as NASH and satisfied the inclusion criteria will be enrolled in the study. Additional subjects may be added on to cover the dropout during the trial. The subjects will be randomly assigned into two groups with a 1:1 randomization ratio ( $\mathrm{n}=80$ for each). For the "control group", the NASH human subjects will be subjected to standardized care and liver protective medication, such as silibinin, polyene phosphatidycholine, and 
diisopropylamine dichloroacetate et al. are often subscripted. For the "VD group", in addition to the standardized protective treatment, vitamin D2 will be administered with an initial dose of $600,000 \mathrm{IU}$ through intramuscular injection, followed by three boosts of 300,000 IU at day 30, day 60, and day 90 . Blood and fecal samples will be collected at the four time points and patients will be subjected to physical assessments, measurements for liver functions, metabolic panel, and fatty liver scores (Table-1).

\section{Intervention safety}

The dosage of vitamin $D_{2}$ used in the trial is based on the Chinese Pharmacopoeia (2015) and approved by the Institutional Review Board, the Public Health and Clinical Center of Chengdu. The safety of mega dose vitamin $D$ through intramuscular injection was previously used by others. For instance, one report showed that 600,000 IU was given to health volunteers with VD deficiency by intramuscular injection, and about $40 \%$ of the human subjects could reach the $25(\mathrm{OH}) \mathrm{VD} 3$ at sufficient levels after eight weeks of administration[18]. Another study showed that injection of $600,000 \mathrm{IU}$ might retain the $25 \mathrm{OH}-\mathrm{VD}_{3}$ at sufficient levels up to four months while mild hypercalcaemia was also observed [19]. To further assess the safety issue of the mega dose $\mathrm{VD}_{2}$ we tested that by injection of mice with ten times of the mega dose of $\mathrm{VD}_{2}$ being used for this trial, by which we did not notice any visible liver injury in histological examination, while moderate hypercalcaemia was noticed.

\section{Criteria for discontinuing or modifying allocated interventions $\{11 \mathrm{~b}\}$}

When any adverse reactions occur, the intervention can be stopped. And during the intervention, participants can choose to stop for any reason of their own.

\section{Outcomes $\{12\}$}

The primary outcomes and secondary outcomes are listed in Table-1.

And the liver fat content will be calculated based on liver ultrasound. In the ultrasonic image, steatosis levels are related to the enhanced near-field echo signal and weakened far-field echo signal. The echo ratio of liver will be measured by specific graphic analysis software (Image J), and the fat content in liver will be calculated by the following two estimation formulas:

Formula \#1: liver fat content = [(73.624) x (standardized liver kidney echo ratio) $]-35.808$;

Formula \#2: liver fat content $=[(62.592) \times($ standardized liver kidney echo ratio $)+(168.076) \times$ (standardized liver echo attenuation rate)] -30.863

\section{Sample size $\{14\}$}

Required sample size was estimated based on formulation[17]. Level of significance $=5 \%$, Power $=80 \%$, Type of test $=$ two-sided. Formula of calculating sample size is $n=\left[\left(Z_{\alpha / 2}+Z_{\beta}\right)^{2} \times\left\{2(y)^{2}\right\}\right] /(\mu 1-\mu 2)^{2}$ 
where

$\mathrm{n}=$ sample size required in each group

$\mu 1=$ mean change in outcome score from baseline to the end in the control group $=5$

$\mu 2=$ mean change in outcome scores from baseline to the end in the VD treatment group $=4.45$

$\mu 1-\mu 2=$ clinically significant difference $=0.5$

$y=$ standard deviation $=1.195$

$Z_{a / 2}:$ This depends on level of significance, for $5 \%$ this is 1.96

$Z_{\beta}$ : This depends on power, for $80 \%$ this is 0.84

Based on the above formula, the sample size required per group is 75 for each group. Considering a dropout rate of $8 \%$ total sample, the size required is 80 in each arm.

\section{Recruitment \{15\}}

The hospital's researchers are responsible for identifying patients who meet the eligibility criteria from both outpatient and inpatient groups. A letter of introduction and "informed consent" will be read to the patients. If patients are willing to participate, they will be randomized into the control group or the VD treatment group. In the trail period and follow-up, we will inform the patients one week in advance and arrange the appointment.

\section{Methods: Assignment of interventions}

\section{Sequence generation $\{16 \mathrm{a}\}$}

Patients who agree and sign the informed consent will be subjected to a computer-generated randomization and assign to the VD treatment group or the control group.

\section{Blinding \{17a\}}

Due to the nature of the intervention, participants and the research team delivering the intervention will not be blinded to the treatment received. Those involved in the data analyses and statistics will be blinded to the group allocation.

\section{Methods: data collection, management, and analysis}

\section{Plans for assessment and collection of outcomes $\{18 \mathrm{a}\}$}

Data will be collected at baseline, 30 days, 60 days, 90 days and 120 days. And the schedule of enrolment, interventions and assessments is provided in Fig 2. 


\section{Data management $\{19\}$}

All participants will receive a trial number that will be used for all of the participant's case report forms. The data will be collected in the safety trial database by hospital researchers.

\section{Statistical analysis $\{20 \mathrm{a}\}$}

Continuous variables characterizing each study group will be expressed as means with standard deviations. Student's $t$ test or two-way ANOVA test will be used for group comparisons. Response to therapy will be evaluated by comparing the values before and after therapy by the paired Student's $t$ test. And the strength of association between quantitative variables will be determined by Pearson's product movement correlation. All statistical measurements will be expressed in terms of $95 \%$ confidence intervals $(\mathrm{Cl})$. Data will be analyzed using the statistical program SPSS version 20.0.

Table. 1 Schedule of enrolment, interventions, and assessments for囚Vitamin $D$ through balancing gut microbiota to improve non-alcoholic steatohepatitis】 


\begin{tabular}{|c|c|c|c|c|c|c|}
\hline \multirow[b]{3}{*}{ TIMEPOINT } & \multicolumn{6}{|c|}{ STUDY PERIOD } \\
\hline & \multirow{2}{*}{$\begin{array}{l}\text { Enrolment } \\
-t_{1}\end{array}$} & \multirow{2}{*}{$\begin{array}{l}\text { Allocation } \\
t_{1} \\
\text { day } 0\end{array}$} & \multicolumn{3}{|c|}{ Post-allocation } & \multirow{2}{*}{$\begin{array}{l}\text { Close- } \\
\text { out } \\
t_{4} \\
\text { day } \\
120\end{array}$} \\
\hline & & & $\begin{array}{l}t_{1} \\
\text { day } 30\end{array}$ & $\begin{array}{l}t_{2} \\
\text { day } 60\end{array}$ & $\begin{array}{l}\boldsymbol{t}_{\mathbf{3}} \\
\text { day } 90\end{array}$ & \\
\hline \multicolumn{7}{|l|}{ ENROLMENT: } \\
\hline $\begin{array}{l}\text { Eligibility screen } \\
\text { and verbal consent }\end{array}$ & $x$ & & & & & \\
\hline Informed consent & $x$ & & & & & \\
\hline Allocation & & $x$ & & & & \\
\hline \multicolumn{7}{|l|}{ INTERVENTIONS: } \\
\hline $\begin{array}{l}\text { [Intervention } A] \\
V D \text { group } \\
V_{2}+\text { liver protective } \\
\text { medication }\end{array}$ & & $\begin{array}{l}600,000 \text { IU VD } \\
\text { protective medication }\end{array}$ & $\begin{array}{l}30,000 \\
\text { IU } \\
\text { Check } \\
\text { up }\end{array}$ & $\begin{array}{l}30,000 \\
\text { IU } \\
\text { Check } \\
\text { up }\end{array}$ & $\begin{array}{l}30,000 \\
\text { IU } \\
\text { Check } \\
\text { up }\end{array}$ & $\begin{array}{l}\text { Follow- } \\
\text { up }\end{array}$ \\
\hline $\begin{array}{l}\text { [Intervention B] } \\
\text { Control group } \\
\text { liver protective } \\
\text { medication }\end{array}$ & & $\begin{array}{l}\text { Liver protective } \\
\text { medication }\end{array}$ & $\begin{array}{l}\text { Check } \\
\text { up }\end{array}$ & $\begin{array}{l}\text { Check } \\
\text { up }\end{array}$ & $\begin{array}{l}\text { Check } \\
\text { up }\end{array}$ & $\begin{array}{l}\text { Follow- } \\
\text { up }\end{array}$ \\
\hline \multicolumn{7}{|l|}{ ASSESSMENTS: } \\
\hline Liver ultrasound & $x$ & $x$ & & & & $x$ \\
\hline Primary outcome & & $x$ & $x$ & $x$ & $x$ & $x$ \\
\hline Secondary outcome & & $x$ & & & & $x$ \\
\hline Adverse Events & & $x$ & $x$ & $x$ & $x$ & $x$ \\
\hline
\end{tabular}




\section{Discussion}

As terrestrial animal, humans have evolved relying on the sunshine, which initiates the synthesis of vitamin $D_{3}$ in the skin followed by additional hydroxylation into calcitriol, which is vital for metabolic homeostasis in many ways. On the other hand, as human migration to the northern continental together with social-ecological development, we rely on vitamin $D$ as a micronutrient, or supplement of food additive in industrialized food chain. Vitamin D insufficiency and deficiency are overwhelmingly prevalent in many societies worldwide, and the tendency of VD deficiency is related to the pigment of the skin, showing dark skinned people have higher prevalence of VD deficiency. Vitamin D through its nuclear receptor (VDR) regulates gene expression at genome wide scale. As an immune adjuvant, vitamin $D$ is known for suppression of adaptive immunity such as Th1 and Th17 response, while VD signaling can up regulates innate immunity, promoting epithelial tight junctions and mucin synthesis. In particular, our previous work found that VDR is highly expressed in the gut epithelial cells including Paneth cells to regulate alpha-defensins that consequently restrain the overgrowth of gut microbes in the small intestine [20]. Our work also demonstrated that dietary $\mathrm{VD}_{3}$ supplement can sufficiently attenuate highfat-diet induced hepatic steatosis, liver injury, inflammation, and metabolic disorders, in part through induction of Paneth cell alpha-defensins that consequently balance gut microbiome. Conversely, depletion of dietary vitamin D or genetic deficiency of VDR could impair the intestinal innate immunity in the mice, leading to gut dysbiosis and steatosis.

Multiple clinical surveys found that reduced serum vitamin $D$ levels are associated with the risk for NAFLD [21-23]. Hepatic steatosis is tightly associated with central obesity, and one report showed that 25(OH)-VD levels are inversely associated with NASH and fibrosis in children with NAFLD [8]. Strong association between NAFLD and low 25(OH)-VD levels was found in an adult population with normal serum liver enzymes [24]. In the NASH patients, VDR expression on cholangiocytes was inversely correlated with steatosis severity, lobular inflammation, and nonalcoholic fatty liver disease score [25]. However, the same group reported that in a small scale trial, oral supplement of VD3 at 2000 IU/daily for 24 weeks to the T2D patient with steatosis had no obvious improvement [26]. Whether vitamin D supplement can improve T2D and NAFLD is controversial, in part due to medical compliance, dose and duration of the trials. Of note and more often, NAFLD are developed over decades by lifestyle and through multiple hits. Vitamin $D$ deficiency may contribute in part to the varying degree for the pathogenesis of T2D and NAFLD. Based on the clinical association and animal work, we speculate that administration of high dose of VD to NASH patients may mitigate the chronic inflammation, promote the intestinal innate immunity and its integrity, balance the gut microbiome to improve the NASH symptoms. In this study we chose vitamin $D_{2}$ (ergocalciferol, derived from plant), and it is known that $\mathrm{VD}_{2}$ and vitamin $\mathrm{D}_{3}$ (cholecalciferol, from animal) have indistinguishable biological functions, and share identical metabolic conversion pathways.

\section{Trial status}


The date of protocol registration was 2018 Aug. 28, and the registration number is ChiCTR1800017879. Recruitment begin on $05 / 28 / 2018$. At the date of this submission to the TRIALs for publication, approximately $120 \mathrm{NASH}$ patients have been recruited and subjected to the study. We estimate the whole work will be completed in 2021.

\section{Abbreviations}

LDH, lactate dehydrogenase;

ALT, alanine transaminase;

AST, aspartate aminotransferase;

GGT, gamma-glutamyltransferase;

ALP, alkaline phosphatase;

TBA, total bile acids;

ALB, serum albumin;

GLO, liver L-gulonolactone oxidase activity; globin

A/G, albumin/globulin;

CHE, cholinesterase

TBIL, serum total bilirubin;

DBIL, serum direct bilirubin;

IBIL, serum indirect bilirubin;

NEFA, non-esterified fatty acids;

BUN, blood urea nitrogen;

CR, creatinine;

UA, uric acid;

Cys-c (Serum cystatin C)

CHOL, cholesterol;

TG, triglycerides; 
LDL-c, low density lipoprotein-cholesterol;

HDL-c, high density lipoprotein-cholesterol;

PBMC, peripheral blood mononuclear cells;

VDR, vitamin D receptor;

LPS, lipopolysaccharides.

\section{Declarations}

\section{Ethics approval and consent to participate $\{24\}$}

The study protocol was approved by the Medical Ethical Committee of the Public Health and Clinical Center of Chengdu (\# 2018Y001). The informed consent will be obtained from all study participants.

This clinical trial has been registered in Chinese Clinical Trial (Registration number: ChiCTR1800017879), and available at http://www.chictr.org.cn/showproj.aspx?proj=25264

\section{Consent for publication $\{31\}$}

All the authors read and approved the manuscript.

\section{Availability of data and material $\{29\}$}

Data and materials will be published in the coming up publication

and uploaded to the web database.

\section{Competing interests $\{28\}$}

The authors declare no competing interest in this study.

\section{Funding $\{4\}$}

The work was supported by Sichuan Science and Technology Program (No. 2017TJPT0013 and 20ZDYF0122) and Public Health and Clinical Center of Chengdu, Scientific Research Project (No.2018K01) to YLZ, and Natural Science Foundation of China (NSFC), \#31571165 and \#31771288 to YPH. The funding agencies did not directly participate in the design of the study and collection, analysis, and interpretation of data and in writing the manuscript.

\section{Authors' contributions $\{31\}$}

YZ, DL, LW, design the study; ML, CM, LZ, RH, BW, HJ, DL, JL, and QL did patient care and collected data; SX, PW, RZ and LP analyzed the data; MN and SLP revised the manuscript; YH drafted the manuscript. 
Acknowledgements

We thank all the members participating this trial in the Public Health and Clinical Center of Chengdu. The authors would like to thank all of the participants who dedicated their time and efforts to participating in the study.

\section{References}

1. Younossi Z, Anstee QM, Marietti M, Hardy T, Henry L, Eslam M, George J, Bugianesi E: Global burden of NAFLD and NASH: trends, predictions, risk factors and prevention. Nat Rev Gastroenterol Hepato/ 2018, 15(1):11-20.

2. Younossi ZM, Koenig AB, Abdelatif D, Fazel Y, Henry L, Wymer M: Global epidemiology of nonalcoholic fatty liver disease-Meta-analytic assessment of prevalence, incidence, and outcomes. Hepatology 2016, 64(1):73-84.

3. Fan JG: Epidemiology of alcoholic and nonalcoholic fatty liver disease in China. Journal of gastroenterology and hepatology 2013, 28 Suppl 1:11-17.

4. Hossein-nezhad A, Spira A, Holick MF: Influence of vitamin D status and vitamin D3 supplementation on genome wide expression of white blood cells: a randomized double-blind clinical trial. PLoS One 2013, 8(3):e58726.

5. Yang BB, Chen YH, Zhang C, Shi CE, Hu KF, Zhou J, Xu DX, Chen X: Low vitamin D status is associated with advanced liver fibrosis in patients with nonalcoholic fatty liver disease. Endocrine 2017, 55(2):582-590.

6. Cordeiro A, Pereira S, Saboya CJ, Ramalho A: Relationship between Nonalcoholic Fatty Liver Disease and Vitamin D Nutritional Status in Extreme Obesity. Can J Gastroenterol Hepatol 2017, 2017:9456897.

7. Park D, Kwon H, Oh SW, Joh HK, Hwang SS, Park JH, Yun JM, Lee H, Chung GE, Ze S et al: Is Vitamin D an Independent Risk Factor of Nonalcoholic Fatty Liver Disease?: a Cross-Sectional Study of the Healthy Population. J Korean Med Sci 2017, 32(1):95-101.

8. Nobili V, Giorgio V, Liccardo D, Bedogni G, Morino G, Alisi A, Cianfarani S: Vitamin D levels and liver histological alterations in children with nonalcoholic fatty liver disease. Eur J Endocrinol 2014, 170(4):547-553.

9. Yodoshi T, Orkin S, Arce-Clachar AC, Bramlage K, Liu C, Fei L, El-Khider F, Dasarathy S, Xanthakos SA, Mouzaki M: Vitamin D deficiency: prevalence and association with liver disease severity in pediatric nonalcoholic fatty liver disease. European journal of clinical nutrition 2019. 
10. Ha Y, Hwang SG, Rim KS: The Association between Vitamin D Insufficiency and Nonalcoholic Fatty Liver Disease: A Population-Based Study. Nutrients 2017, 9(8).

11. Li L, Zhang L, Pan S, Wu X, Yin X: No significant association between vitamin D and nonalcoholic fatty liver disease in a Chinese population. Dig Dis Sci 2013, 58(8):2376-2382.

12. Jaruvongvanich V, Ahuja W, Sanguankeo A, Wijarnpreecha K, Upala S: Vitamin D and histologic severity of nonalcoholic fatty liver disease: A systematic review and meta-analysis. Digestive and liver disease : official journal of the Italian Society of Gastroenterology and the Italian Association for the Study of the Liver 2017, 49(6):618-622.

13. Zhu S, Wang Y, Luo F, Liu J, Xiu L, Qin J, Wang T, Yu N, Wu H, Zou T: The Level of Vitamin D in Children and Adolescents with Nonalcoholic Fatty Liver Disease: A Meta-Analysis. BioMed research international 2019, 2019:7643542.

14. Tukvadze N, Sanikidze E, Kipiani M, Hebbar G, Easley KA, Shenvi N, Kempker RR, Frediani JK, Mirtskhulava V, Alvarez JA et al: High-dose vitamin D3 in adults with pulmonary tuberculosis: a doubleblind randomized controlled trial. Am J Clin Nutr 2015, 102(5):1059-1069.

15. Tangpricha V, Smith EM, Binongo J, Judd SE, Ziegler TR, Walker S, Tirouvanziam R, Zughaier SM, Lee MJ, Chesdachai S et al: The Vitamin D for Enhancing the Immune System in Cystic Fibrosis (DISC) trial: Rationale and design of a multi-center, double-blind, placebo-controlled trial of high dose bolus administration of vitamin D3 during acute pulmonary exacerbation of cystic fibrosis. Contemp Clin Trials Commun 2017, 6:39-45.

16. Narula N, Cooray M, Anglin R, Muqtadir Z, Narula A, Marshall JK: Impact of High-Dose Vitamin D3 Supplementation in Patients with Crohn's Disease in Remission: A Pilot Randomized Double-Blind Controlled Study. Dig Dis Sci 2017, 62(2):448-455.

17. Sakpal TV: Sample size estimation in clinical trial. Perspect Clin Res 2010, 1(2):67-69.

18. Khan AH, Rohra DK, Saghir SA, Udani SK, Wood R, Jabbar A: Response of a single 'mega intramuscular dose' of vitamin D on serum 250HD and parathyroid hormone levels. J Coll Physicians Surg Pak 2012, 22(4):207-212.

19. Diamond TH, Ho KW, Rohl PG, Meerkin M: Annual intramuscular injection of a megadose of cholecalciferol for treatment of vitamin D deficiency: efficacy and safety data. Med J Aust 2005, 183(1):10-12.

20. Su D, Nie Y, Zhu A, Chen Z, Wu P, Zhang L, Luo M, Sun Q, Cai L, Lai Y et al: Vitamin D Signaling through Induction of Paneth Cell Defensins Maintains Gut Microbiota and Improves Metabolic Disorders and Hepatic Steatosis in Animal Models. Front Physio/ 2016, 7:498. 
21. Rhee EJ, Kim MK, Park SE, Park CY, Baek KH, Lee WY, Kang MI, Park SW, Kim SW, Oh KW: High serum vitamin $D$ levels reduce the risk for nonalcoholic fatty liver disease in healthy men independent of metabolic syndrome. Endocr J 2013, 60(6):743-752.

22. Lu Z, Pan X, Hu Y, Hao Y, Luo Y, Hu X, Ma X, Bao Y, Jia W: Serum vitamin D levels are inversely related with non-alcoholic fatty liver disease independent of visceral obesity in Chinese postmenopausal women. Clin Exp Pharmacol Physiol 2015, 42(2):139-145.

23. Wang D, Lin H, Xia M, Aleteng Q, Li X, Ma H, Pan B, Gao J, Gao X: Vitamin D Levels Are Inversely Associated with Liver Fat Content and Risk of Non-Alcoholic Fatty Liver Disease in a Chinese Middle-Aged and Elderly Population: The Shanghai Changfeng Study. PLoS One 2016, 11(6):e0157515.

24. Barchetta I, Angelico F, Del Ben M, Baroni MG, Pozzilli P, Morini S, Cavallo MG: Strong association between non alcoholic fatty liver disease (NAFLD) and low 25(OH) vitamin D levels in an adult population with normal serum liver enzymes. BMC Med 2011, 9:85.

25. Barchetta I, Carotti S, Labbadia G, Gentilucci UV, Muda AO, Angelico F, Silecchia G, Leonetti F, Fraioli A, Picardi A et al: Liver vitamin D receptor, CYP2R1, and CYP27A1 expression: relationship with liver histology and vitamin D3 levels in patients with nonalcoholic steatohepatitis or hepatitis C virus. Hepatology 2012, 56(6):2180-2187.

26. Barchetta I, Del Ben M, Angelico F, Di Martino M, Fraioli A, La Torre G, Saulle R, Perri L, Morini S, Tiberti $C$ et al: No effects of oral vitamin D supplementation on non-alcoholic fatty liver disease in patients with type 2 diabetes: a randomized, double-blind, placebo-controlled trial. BMC Med 2016, 14:92.

\section{Figures}


Figure 1

A

Association study, NASH patients

2. Gut microbiome;

3. Liver functions;

4. Metabolic panel;

5. Fatty liver imaging scores.

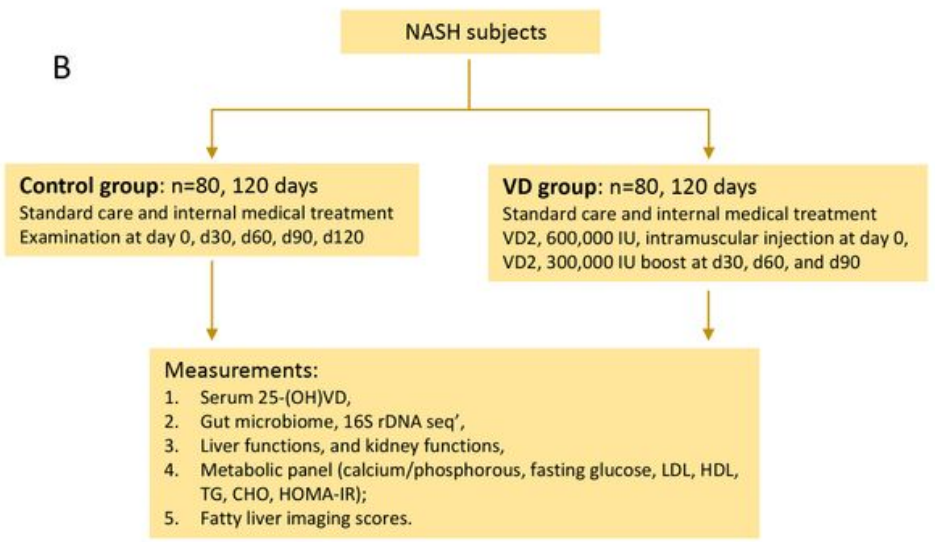

\section{Figure 1}

Flowchart of the study. NASH patients are diagnosed according to the Guidelines for NFALD (Chinese Society of Hepatology, 2015). The patients who satisfied the enrollment criteria will be randomized into control and VD treatment group. The anticipated sample size is estimated, $n=80$ for each group. The care and treatment for the two groups are described in the Methods and Design. (A) Association of vitamin D deficiency with the parameters of NAFLD and gut microbiota. (B) Therapeutic efficacy and impact on gut microbiomes by high dose of vitamin D2 given to NASH patients. For the VD treatment group, an initial dose of 600,000 IU of VD2 are given by intramuscular injection (Chinese pharmacopoeia, 2015), followed by three boosts, at 300,000 IU each with interval of 33 days, at day 30,60 , and 90 respectively. The last measurement is scheduled at d30ay 120 . 